\title{
ON MAPPINGS CONTRACTIVE IN THE SENSE OF KANNAN
}

\author{
LUDVIK JANOS
}

\begin{abstract}
Let $f: X \rightarrow X$ be a continuous compact mapping of a metric space $(X, d)$ into itself with the property that $x, y \in X$ and $x \neq y$ implies $d(f(x), f(y))<\frac{1}{2}[d(x, f(x))+d(y, f(y))]$. It is shown that under these conditions $f$ has a unique fixed point and, moreover, $f$ is a Banach contraction relative to a suitable remetrization of the space $X$. A similar result concerning condensing mappings is also obtained.
\end{abstract}

1. Introduction. A fixed point theorem for a certain class of metric spaces $(X, d)$ (e.g. complete, bounded, compact etc.) is an assertion about a self-map $f: X \rightarrow X$ satisfying certain conditions, some of which may be of topological nature (e.g. continuity), and others involving explicitly the metric $d$. The purpose of this note is to investigate a relation between the classical Banach contraction principle and its generalizations by Kannan (see [4] and [5]) and Edelstein ([2] and [1]). The pertaining metric conditions mentioned above are the three types of contraction inequalities contained in the following definitions: A self-map $f: X \rightarrow X$ of a metric space $(X, d)$ is said to be a contraction (in the sense of Banach) if there exists $c \in(0,1)$ (the Lipschitz constant) such that whenever $x$ and $y$ belong to $X, d(f(x), f(y)) \leqslant c d(x, y)$. Similarly, we say that $f$ is Kannan contractive if $x \neq y$ implies

$$
d(f(x), f(y))<\frac{1}{2}[d(x, f(x))+d(y, f(y))]
$$

and Edelstein contractive if $x \neq y$ implies

$$
d(f(x), f(y))<d(x, y) .
$$

In his paper Evidence of a conspiracy among fixed point theorems [8], Ira Rosenholtz observed that one kind of contractivity relative to a metric $d$ may imply another kind relative to a suitable metric $d^{*}$ topologically equivalent to $d$. Following that idea, we first focus our attention on the relation between Kannan-contractivity and the Banach contraction property. We denote by $M(X)$ the set of all metrics on $X$ that are topologically equivalent to $d$ for a given metric space $(X, d)$. Our main result reads as follows:

Received by the editors February 6, 1976 and, in revised form, March 18, 1976.

AMS (MOS) subject classifications (1970). Primary 54H25, 54E40; Secondary 54E35, 54E45.

Key words and phrases. Fixed points, Banach contraction principle, contractions, self-maps contractive in the sense of Kannan and Edelstein, remetrization, Kuratowski's and Hausdorff's measures of noncompactness, condensing mapping. 
TheOrem 1.1. Let $f: X \rightarrow X$ be a Kannan contractive continuous and compact mapping of a metric space $(X, d)$ into itself. Then $f$ has a unique fixed point, and, furthermore, for any $c \in(0,1)$ there exists a metric $d^{*} \in M(X)$ relative to which $f$ is a contraction with Lipschitz constant $c$.

2. Proof of the theorem. By our assumption that $f$ is a compact map there exists a compact subset $Y$ of $X$ containing $f(X)$, and we observe that $f(Y) \subset Y$. This in turn implies that the set $A$ defined as the intersection $\bigcap_{n=1}^{\infty} f^{n}(Y)$ of the iterative images $f^{n}(Y)$ is a nonempty compact $f$-invariant subset of $X$ which is mapped by $f$ onto itself. We now show that $A$ is a onepoint set, i.e., $A=\{a\}$ for some $a \in X$ which is the unique fixed point of $f$. If this were not the case then the diameter $d=d(A)$ of $A$ would be a positive number $d>0$. Due to the compactness of $A$ there would exist points $x, y \in A$ with $d(x, y)=d$ and due to the fact that $f$ maps $A$ onto itself there would exist points $x^{*}, y^{*} \in A$ with $x=f\left(x^{*}\right)$ and $y=f\left(y^{*}\right)$. Applying to these points the Kannan inequality we obtain

$$
d=d(x, y)=d\left[f\left(x^{*}\right), f\left(y^{*}\right)\right]<\frac{1}{2}\left[d\left(x^{*}, x\right)+d\left(y^{*}, y\right)\right] \leqslant d
$$

which is the desired contradiction, proving that $f$ has a unique fixed point $a$.

In order to prove the rest of Theorem 1.1 we need the following result of $P$. R. Meyers [7]:

TheOrem 2.1 (P. R. Meyers). Let $f: X \rightarrow X$ be a continuous self-map of $a$ metric space $(X, d)$ into itself with the following properties:

(i) f has a unique fixed point $a \in X$.

(ii) For every $x \in X$ the sequence of iterations $\left\{f^{n}(x)\right\}$ converges to a.

(iii) There exists an open neighborhood $U$ of a with the property that given any open set $V$ containing a there exists an integer $n_{0}$ such that $n \geqslant n_{0}$ implies $f^{n}(U) \subset V$.

Then for an arbitrary $c \in(0,1)$ there exists a metric $d^{*} \in M(X)$ relative to which $f$ is a contraction with the Lipschitz constant $c$.

We now verify readily that our map $f$ satisfies all of these three conditions of Theorem 2.1. In fact, (i) has already been proved, and (ii) follows from the fact that for any $x \in X$ the sequence $\left\{f^{n}(x)\right\}$ is contained in $Y$. To show that $f$ also satisfies (iii) we take $U=X$ and observe that $f^{n+1}(X)$ is contained in $f^{n}(Y)$, the diameter of which diminishes to zero as $n \rightarrow \infty$. Thus $f^{n}(X)$ squeezes into any neighborhood of $a$ and the proof of Theorem 1.1 is complete.

REMARK. Theorem 1.1 remains valid if the Kannan contractivity appearing in its hypothesis is replaced by some other types of contractivity, for example, as introduced by

$$
x \neq y \Rightarrow d(f(x), f(y))<\frac{1}{2}[d(x, f(y))+d(y, f(x))]
$$

or by 


$$
x \neq y \Rightarrow d(f(x), f(y))<\frac{1}{3}[d(x, f(x))+d(x, y)+d(y, f(y))]
$$

since the argument in the proof of this theorem concerning the set $A$ $=\bigcap_{n=1}^{\infty} f^{n}(Y)$ remains valid in these cases.

What we have just proved means that the Kannan contractivity implies under certain additional conditions and under a suitable remetrization of the space $X$ the Banach contraction property. We now show that the converse is also true.

Lemma 2.2. Let $f: X \rightarrow X$ be a contraction of a metric space $(X, d)$ with the Lipschitz constant $c<\frac{1}{3}$. Then $f$ is Kannan contractive relative to $d$.

Proof. Let $x, y \in X$. From the triangle inequality we obtain

$$
d(f(x), f(y)) \leqslant(c /(1-c))[d(x, f(x))+d(y, f(y))]
$$

which combined with the contraction inequality $d(f(x), f(y)) \leqslant c d(x, y)$ yields

$$
d(f(x), f(y)) \leqslant(c / 1-c))[d(x, f(x))+d(y, f(y))]
$$

from which our assertion follows.

TheOREM 2.3. Let $f: X \rightarrow X$ be a contraction of a metric space $(X, d)$ having a fixed point $a \in X$. (We do not postulate $(X, d)$ to be complete.) Then there exists a metric $d^{*} \in M(X)$ relative to which $f$ is Kannan contractive.

Proof. Since $f$ has the fixed point $a \in X, f$ satisfies all the requirements of Theorem 2.1. Thus, choosing $c<\frac{1}{3}$ we obtain a new metric $d^{*} \in M(X)$ relative to which $f$ is a contraction with the Lipschitz constant $c<\frac{1}{3}$ which by Lemma 2.2 implies that $f$ is Kannan contractive relative to $d^{*}$.

3. Some generalizations to condensing mappings. Denoting by $\alpha(Y)$ the Kuratowski measure of noncompactness of a subset $Y$ of a metric space $(X, d)$, defined as the infimum of those numbers $\varepsilon>0$ for which $Y$ can be covered by a finite number of sets of diameter $\leqslant \varepsilon$ (see [6] and [9]), we say that a continuous self-map $f: X \rightarrow X$ of a bounded metric space $(X, d)$ is condensing if for any nonempty and nontotally bounded subset $Y$ of $X \alpha(f(Y))<\alpha(Y)$ holds. We note that for our purposes the Kuratowski measure of noncompactness can be replaced by that of Hausdorff (see [9]). We now show that if the compactness condition in Theorem 1.1 is relaxed, requiring only the mapping $f$ to be condensing, and imposing at the same time the condition of completeness on the space $(X, d)$, then the mapping $f$ has a unique fixed point and is Edelstein contractive relative to a suitable metric $d^{*} \in M(X)$. To prove this statement we need the following result on Edelstein contractive mappings proved in [3]. 
THEOREM 3.1. Let $X$ be a metrizable topological space and $f: X \rightarrow X$ a continuous map such that the sequence $\left\{f^{n}(x)\right\}$ converges for every $x \in X$. Then the following two statements are equivalent:

(i) There is a metric $d \in M(X)$ such that $f$ is Edelstein contractive relative to $d$.

(ii) For every nonempty compact f-invariant subset $Y$ of $X$ the intersection $\cap_{n=1}^{\infty} f^{n}(Y)$ is a one-point set.

We are now ready to prove

THEOREM 3.2. Let $f$ be a condensing Kannan self-map of a complete bounded metric space $(X, d)$. Then $f$ has a unique fixed point and there is a metric $d^{*} \in M(X)$ relative to which $f$ is Edelstein contractive.

Proof. If $x \in X$ we denote by $\Gamma(x)$ the closure of its orbit $O(x)$ $=\left\{x, f(x), f^{2}(x), \ldots\right\}$. Due to the completeness of $(X, d)$ and the obvious fact that $\alpha[O(f(x))]=\alpha[O(x)]$, we conclude that $\Gamma(x)$ is compact. Since $\Gamma(x)$ is $f$ invariant we can apply Theorem 1.1 to it obtaining that the sequence $\left\{f^{n}(x)\right\}$ converges to the unique fixed point of $f$. Thus the hypothesis of Theorem 3.1 is satisfied by $f$ and all we need is to verify that condition (ii) in that theorem is also satisfied. But this follows by the same argument (using the Kannan contractivity of $f$ ) as used in the proof of Theorem 1.1 when showing that $\cap_{n=1}^{\infty} f^{n}(Y)$ is a one-point set, and our assertion follows.

REMARK. We do not know so far whether Theorem 3.2 has a converse in the sense that the contractivity conditions in the sense of Kannan and Edelstein appearing in its hypothesis and conclusion can interchange their rôles. Also we do not know whether the conclusion of this theorem can be strengthened claiming that $f$ is a contraction relative to some metric $d^{*} \in M(X)$. However, assuming $(X, d)$ compact and $f: X \rightarrow X$ continuous we obtain as a corollary of Theorems 1.1, 2.1, 2.3, and 3.1 the equivalence of the following statements:

(i) $f$ is Kannan contractive relative to some $d^{*} \in M(X)$.

(ii) $f$ is Edelstein contractive relative to some $d^{*} \in M(X)$.

(iii) Given $c \in(0,1)$ there is $d^{*} \in M(X)$ relative to which $f$ is a contraction with Lipschitz constant $c$.

\section{REFERENCES}

1. J. Bryant and L. F. Guseman, Jr., Extensions of contractive mappings and Edelstein's iterative test, Canad. Math. Bull. 16 (1973), 185-192. MR 48 \#2844.

2. M. Edelstein, On fixed and periodic points under contractive mappings, J. London Math. Soc. 37 (1962), 74-79. MR 24 \# A2936.

3. L. Janos, On the Edelstein contractive mapping theorem, Canad. Math. Bull. 18 (1975), 675-678.

4. R. Kannan, Some results on fixed points, Bull. Calcutta Math. Soc. 60 (1968), 71-76. MR 41 \#2486.

5. - Some results on fixed points. II, Amer. Math. Monthly 76 (1969), 405-408. MR 41 $\# 2487$.

6. K. Kuratowski, Sur les espaces complets, Fund. Math. 15 (1930), 301-309.

7. P. R. Meyers, A converse to Banach's contraction theorem, J. Res. Nat. Bur. Standards Sect. B 71B (1967), 73-76. MR 36 \#4521. 
8. Ira Rosenholtz, Evidence of a conspiracy among fixed point theorems, Proc. Amer. Math. Soc. 53 (1975), 213-218.

9. B. N. Sadovskiī, Limit-compact and condensing operators, Uspehi Mat. Nauk 27 (1972), no. 1 (163), 81-146 = Russian Math. Surveys 27 (1972), no. 1, 85-155.

Department of Mathematics, Washington State University, Pullman, Washington 99163

Current address: Department of Mathematics, Mississippi State University, Mississippi State, Mississippi 39762 